\title{
Double unification of particles with fields and electricity with gravity in non-empty space of continuous complex energies
}

\author{
Igor E. Bulyzhenkov ${ }^{1,2,3, a}$ \\ ${ }^{1}$ Moscow Institute of Physics and Technology, 9 Institutskiy per., Dolgoprudny, Moscow reg., 141700, Russia \\ ${ }^{2}$ National Science and Technology University MISiS, 4 Leninsky pros., Moscow, 119049, Russia \\ ${ }^{3}$ Lebedev Physics Institute RAS, 53 Lininsky pros., Moscow, 119991, Russia
}

\begin{abstract}
Non-empty space reading of Maxwell equations as local energy identities explains why a Coulomb field is carried rigidly by electrons in experiments. The analytical solution of the Poisson equation defines the sharp radial shape of charged elementary densities which are proportional to continuous densities of electric self-energy. Both Coulomb field and radial charge densities are free from energy divergences. Non-empty space of electrically charged mass-energy can be described by complex analytical densities resulting in real values for volume mass integrals and in imaginary values for volume charge integrals. Imaginary electric charges in the Newton gravitational law comply with real Coulomb forces. Unification of forces through complex charges rids them of radiation self-acceleration. Strong gravitational fields repeal probe bodies that might explain the accelerated expansion of the dense Metagalaxy. Outward and inward spherical waves form the standing wave process within the radial carrier of complex energy.
\end{abstract}

\section{Introduction}

Recent measurements of dynamical electric fields associated with moving charges "support the idea of a Coulomb field carried rigidly by the electron beam" [1]. This experimental result seems natural only in non-empty space physics, where charged radial fields represent distributions of continuous elementary matter in the very radial structure of its Coulomb/Newton fields. Non-empty space (inferred by Plato and Aristotle as a continuous material plenum beyond observations) can be better justified through extended electric charges in the laboratory, rather than through extended masses in observed weak gravitation. Since the days of the ancient Greeks, non-empty space for physical reality had been repeatedly claimed by many thinkers, including Einstein: "A coherent field theory requires that all elements be continuous... And from this requirement arises the fact that the material particle has no place as a basic concept in a field theory. Thus, even apart from the fact that it does not include gravitation, Maxwell's theory cannot be considered as a complete theory" (translation [2]). In this paper we try to comment quantitatively on this Einstein's vision of a coherent theory of pure classical fields which could indeed strike out the particle as an independent concept.

The postulated point-particle paradigm results not only in the mathematical divergence of the Coulomb energy, but also in the physical inconsistence of the microscopic electron theory. Any

ae-mail: bulyzhenkov.ie@mipt.ru 


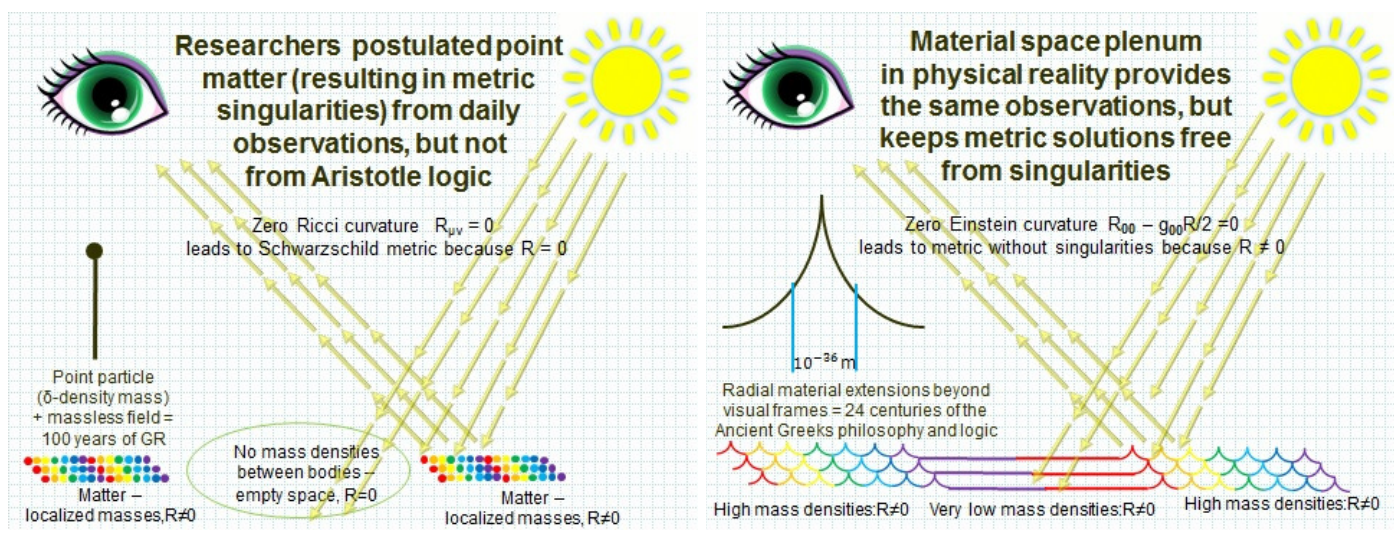

Figure 1. Empty space and point particles vs non-empty space physics of radial energy carries

point source in the microscopic Maxwell-Lorentz equations may be considered as "an attempt which we have called intellectually unsatisfying" according to De Broglie [3]. Einstein also criticized his 1915 field equation for the point gravitational source: "it resembles a building with one wing built of resplendent marble and the other built of cheap wood" (translation [2]). The Dirac delta-operator formalism for the point charge seems a provisional modeling of physical reality until local analytical charge-field relations can be finally proposed for Maxwell's electrodynamics and Einstein's gravitation. Recall that modern researchers postulate point matter (resulting in metric singularities) from daily observations, but not from analytical mathematics or logic. The latter had lead Aristotle (after Plato's aether and material forms) to the conclusion that space is to be a continuous material plenum without empty regions (material bodied cannot be placed to such empty regions without logical inconsistencies). Non-dual physical reality of non-empty space with extremely low and extremely high mass-energy densities provides the same observations in practice as the dual (massive particle + massless field) physical model, but keeps metric solutions free from singularities (see comments on the Figure 1). Indeed, the scalar Ricci curvature $R=g^{\mu v} R_{\mu v}$ is proportional to a scalar mass density in non-empty 3 -space and, therefore, $R \neq 0$ in all space-time points. From here, static metric in non-dual reality of inhomogeneous mass-energy densities is to be defined by a zero Einstein curvature $R_{o}^{o}-R / 2=0$, rather than by a zero Ricci curvature $R_{o}^{o}=0$ for the conventional dual model in question.

As to non-empty space for the electromagnetic domain, a continuously distributed elementary charge was reasonably inferred by Mie in order to derive properties of charges from properties of fields (and potentials) and to overcome the energy divergence flaw in the Coulomb field center. Regretfully, in 1912-1913 and later the 'Theory der Matter' [4] had not found the appropriate (logarithmic) post-Coulomb potential for strong fields. And the promising non-empty space concept had not been justified before the mathematical era of quantum non-locality. The formal probabilities for the delta-operator 'dice' in the same empty space arena postponed the search of rigorous analytical solutions for Mie's nonlocal matter. In 1984 Schwinger intuitively proposed [5] to extend the point classical electron by the exponential radial distribution like in the Yukawa field potential. However, the annoying problem of the unphysical point source and the Coulomb energy divergence are still unresolved satisfactorily within the trustful mathematical formalism of classical fields. These permanent challenges of contemporary theoretical and mathematical physics may be considered as a motivation 
for our reinforcement of Mie and Schwinger nonlocal (astro)particle by the continuous radial density over the infinite world volume or the entire Universe.

\section{Radial densities of imaginary electric charges}

Let us revisit classical electromagnetic equations regarding coherent solutions for a distributed particle and its field. By following Mie, we assume that it is possible to relate analytically electric, $\vec{d}(x)$, and magnetic, $\vec{b}(x)$, field intensities in the Maxwell-Lorentz equations [6],

$$
\left\{\begin{array}{l}
\operatorname{div} \vec{d}(\mathbf{x}, t)=4 \pi \rho(\mathbf{x}, t) \\
\operatorname{div} \vec{b}(\mathbf{x}, t)=0 \\
\operatorname{ccurl} \vec{b}(\mathbf{x}, t)=4 \pi \rho(\mathbf{x}, t) \vec{v}+\partial_{t} \vec{d}(\mathbf{x}, t) \\
\operatorname{ccurl} \vec{d}(\mathbf{x}, t)=-\partial_{t} \vec{b}(\mathbf{x}, t),
\end{array}\right.
$$

to the local charge, $\rho(\mathbf{x}, t)$, and current, $\rho(\mathbf{x}, t) \vec{v}$, densities of the extended electron. Contrary to Lorentz, the electron's charge distribution was not postulated by Mie within a microscopic spatial volume (electron's fields were assigned by Lorentz to charge-free regions or to the supposed empty space around charges). The mathematical equation $\operatorname{div} \vec{d}(\mathbf{x}, t)=4 \pi \rho(\mathbf{x}, t)$ can be rigorously resolved under the non-empty space assumption by admitting that the elementary charge and its field coexist together in all space points $\mathbf{x}$ of the infinite Universe. In other words, we tend to maintain the idea of Mie (and Einstein) that the elementary electric (and gravitational) charge is to be integrated into its spatial field structure with instantaneous local relations between scalar functions $\rho(\mathbf{x}, t)$ and $\vec{d}(\mathbf{x}, t) \cdot \vec{d}(\mathbf{x}, t)$. Then locally paired, rigid motion of Coulomb radial fields and electron's radial shapes can maintain the precision experiment [1] as a direct justification of the non-empty space paradigm for physical reality.

The mass density $m_{o} n(r)$ of the distributed radial electron with the analytical density $n(r)$ possesses the same active mass-energy density as the passive mass-energy of electron's gravitational field (due to the Principle of Equivalence). Similarly, the charge density $\rho(r)=q n(r)$ of the same electron should possess a self-energy density which is to be equal to the Coulomb field energy density. Therefore, the passive charge density of the extended particle is to be proportional to the active field energy density

$$
\rho(\mathbf{x}, t)=[ \pm \vec{d}(\mathbf{x}, t)]^{2} / 4 \pi \varphi_{o}
$$

in the rest frame of reference. Such physical relations between Coulomb's field intensity $\vec{d}(\mathbf{x}, t)$ and particle's density $\rho(\mathbf{x}, t)$ can be expected [7] among correct solutions to Maxwell's equations if the Einstein Principle of Equivalence works for electricity. Moreover, the electric charge $q$, with selfenergy $q \varphi_{o}$, and its density $\rho$, with self-energy density $\rho \varphi_{o}$, has the energy nature under the universal potential $\varphi_{o}=$ const like a mass has the energy nature due to $E=m c^{2}$. Maxwell-Lorentz's equations can be equally discussed as for electric currents with the electric density $\rho$ as well as for energy flows with the electric energy density $\rho \varphi_{o}$. In general, electric self-energy $E_{q}=q \varphi_{o}$ of the charge $q$ should be added to the mechanical self-energy $E_{m}=m c^{2}$ of a charged body.

One can say that EM energy flows with their natural conservations/dissipations are more fundamental for unification of electrodynamics and thermodynamics than electric currents with the well defined conservation of charges but ill-defined dissipation notions. Anyway, classical electrodynamics can fluently employ the charge self-energy (justified so far only in quantum electrodynamics) in Mie's non-empty space with material fields. Recall, that these fields can represent continuous particlecharges. The delta-operator description of localized charges, say on separated capacitor's plates, can also introduce the electrostatic energy of such point charges in addition to the equal amount of 
their Coulomb field energy (separated spatially from such point charges). Contrary to diverging selfenergies of point particles, self-energy of the spatially distributed charge can be finite and can be described through analytical densities with the clear physical meaning.

The local equality of the charge energy density, $\rho \varphi_{o}$, and the Coulomb field energy density, $\vec{d}^{2} / 4 \pi$, assumes finite electrostatic energy integrals of an elementary continuous carrier of electricity,

$$
\int \frac{\vec{d}^{2}(\mathbf{x}, t)}{4 \pi} d v=\varphi_{o} \int \rho(\mathbf{x}, t) d v \equiv \varphi_{o} q \neq \infty .
$$

This divergence-free energy distributions for a continuous elementary carrier 'at rest' $(\vec{v}=\vec{b}=0)$ complies with div $\vec{d}(r)=4 \pi \rho(r)$ under exact radial solutions for field and charge densities,

$$
\left\{\begin{array}{l}
\vec{d}(r)=q \hat{\mathbf{r}} / r\left(r+r_{o}\right) \\
\rho(r)=q r_{o} / 4 \pi r^{2}\left(r+r_{o}\right)^{2},
\end{array}\right.
$$

where the particle's density $n(r)=r_{o} / 4 \pi r^{2}\left(r+r_{o}\right)^{2}$ in non-empty space replicates the Dirac operator density $\delta(r)$ under the empty space approximation of reality. The exact solutions (4) match the finite balance of electric energy (3) and rids Maxwell electrodynamics of the Coulomb energy divergence. Notice that the extended classical particle in (4) is distributed over macro and mega scales (as well as over Planck scales and the quantum microworld) that denies the localized particle.

The field flux of the radial charge distribution $\rho(r)$ depends on a selected radius $\mathrm{R}$ for a Gaussian sphere,

$$
4 \pi R^{2} \hat{\mathbf{r}} \vec{d}(R) \equiv q(R)=\int_{0}^{R} \rho(r) 4 \pi r^{2} d r=\int_{0}^{R} \frac{q(\infty) r_{o} d r}{\left(r+r_{o}\right)^{2}}=q(\infty) \frac{R}{R+r_{o}},
$$

where the complex parameter $r_{o}$ defines the half-charge scale $\left|r_{o}\right|$ for the infinite spatial (astro)distribution (4) of the total charge $q \equiv q(\infty)$. In other words, any non-zero value $r_{o} \neq 0$ unavoidably results in the global astrodistribution of the elementary charge over the entire Universe. Therefore, the non-point Maxwell electron (4) cannot be localized in principle within the microscopic (and even macroscopic) volume assumed in the Lorentz model. The classical charge density is rigidly bound with the electric field energy density, $\rho \propto \overrightarrow{\mathrm{d}}^{2}$. The equivalent charge and its field counter-flows of imaginary electric energies fill non-empty world space continuously together with collinear (residual) co-flows of real mass-energies within the elementary radial carrier [7]. The exact mathematical solution (4) to the classical field equations (1) admits the global material overlap of all elementary charges and rejects the empty space simplification of reality through separated fields and particles.

One can use imagine numbers for the 'negative' elementary charge of the electron, $\int \rho(\mathbf{x}, t) d v \equiv$ $q \equiv i e \Rightarrow-i e_{o}$. The charge self-potential $\varphi_{o} \equiv E_{q} / q=q / r_{o}=c^{2} / \sqrt{G}$ we defined via the light speed limit $c$ and the Cavendish constant $G$. The similar charge to spatial scale ratio, $\sqrt{G} m / r_{m}=c^{2} / \sqrt{G}$, arises in gravitation of continuous masses [7], where $m c^{2} \equiv \sqrt{G} m \varphi_{o}$ is the body relativistic energy. We keep the fundamental potential $\varphi_{o} \equiv c^{2} / \sqrt{G}=3.48 \times 10^{27} \mathrm{Stat} V=1.04 \times 10^{27} \mathrm{~V}$ for self-energies of both mechanical and electric charges,

$$
E=(\sqrt{G} m+q) \varphi_{o}=m c^{2}+i e G^{-1 / 2} c^{2},
$$

in order to extend the Einstein mechanical formula on electricity.

Despite the electron possesses in (6) only imaginary electric energy $\left(-i \cdot 1,04 \times 10^{24} \mathrm{KeV}\right.$ of the imaginary charge $q=-i e_{o}$ ) next to $511 \mathrm{KeV}$ of the real mechanical energy, paired interactions of imaginary electric charges correspond to real Coulomb forces and to real interaction energies. 
Contrary to an electric charge defined in real numbers, like the inertial mass, the imaginary electric charge exhibits a correct direction of the radiation self-force, which is proportional to $q^{2}=-e_{o}^{2}<0$. Ultimately, unified densities of gravito-mechanic and electric charges can be described in complex functions. Real integrals over spatial densities provide masses of energy carriers, imaginary integrals - electric charges.

\section{Logarithmic strong field potential for complex energy carriers}

The strong-field potential $W(\mathbf{x})=-\varphi_{o} \ln \left[1+\sqrt{G} M /|\mathbf{x}| \varphi_{o}\right]$ of the gravitational charge $\sqrt{G} M$ could be introduced [7] for interaction with other gravitational charges $\sqrt{G} m$. This radial potential for static gravitational fields matches zero Einstein curvature, $G_{o}^{o} \equiv R_{o}^{o}-R / 2=0$, and the flat-space subinterval, $\left(g_{o i} g_{o j} g_{o o}^{-1}-g_{i j}\right) d x^{i} d x^{j}=\delta_{i j} d x^{i} d x^{j}$, in the relativistic four-interval:

$$
d s^{2}=\frac{c^{2} d t^{2}}{\left(1+\sqrt{G} M / r \varphi_{o}\right)^{2}}-d r^{2}-r^{2} d \theta^{2}-r^{2} \sin \theta^{2} d \phi^{2}
$$

The mechanical self-energy $\varphi_{o} \sqrt{G} m=m c^{2}$ of the charge $\sqrt{G} m$ arises to compensate the integral self-action of these charge densities $\rho_{m}(\mathbf{x})=\nabla^{2} W(\mathbf{x}) / 4 \pi=m r_{o} / 4 \pi \mathbf{x}^{2}\left(r_{o}+|\mathbf{x}|\right)^{2}$ in its negative interaction potential $W(\mathbf{x})$,

$$
\begin{array}{r}
m c^{2} \equiv \varphi_{o} \sqrt{G} m \equiv \int \varphi_{o} \rho_{m}(\mathbf{x}) d^{3} x=-\int W(\mathbf{x}) \rho_{m}(\mathbf{x}) d^{3} x \text { or } \\
\int d^{3} x\left[\varphi_{o}+W(\mathbf{x})\right] \nabla^{2} W(\mathbf{x}) / 4 \pi=0
\end{array}
$$

This universal compensation of Einstein's mechanical self-energy $m c^{2}$ by the self-interaction of mass densities in negative gravitational potentials takes place due to the integral mathematical equality $\int_{o}^{\infty} d x\left[\ln \left(1+x^{-1}\right)\right] /(1+x)^{2} \equiv \int_{o}^{\infty} d x /(1+x)^{2}=1$.

The gravity-type Poisson equation, $\nabla^{2} W=4 \pi \rho \Rightarrow \varphi_{o}^{-1}(\nabla W)^{2}$, reads the imaginary (astro)electron as a non-linear field composition with respect to the radial field intensity $d(r)$ or the electron's interaction potential $W(r)$, with $d(r)=-\partial_{r} W(r)$ and

$$
\frac{\partial_{r}\left[r^{2} \partial_{r} W(r)\right]}{4 \pi r^{2}}=\frac{\left[\partial_{r} W(r)\right]^{2}}{4 \pi \varphi_{o}}=\frac{q r_{o}}{4 \pi r^{2}\left(r+r_{o}\right)^{2}} .
$$

Such a s non-linear equation reveals the imaginary post-Coulomb potential with the negative, gravitational sign for the continuous radial carrier of electricity,

$$
W(r)=-\frac{q}{r_{o}} \ln \left(1+\frac{r_{o}}{r}\right) \equiv-\varphi_{o} \ln \left(1+\frac{q}{\varphi_{o} r}\right) .
$$

This logarithmic potential reproduces the regular Coulomb law on measurable scales with an opposite, Newtonian sign $\left(-q / r_{o}\right) \ln \left[\left(r+r_{o}\right) / r\right] \approx(-q / r)$, when $\left|r_{o}\right| \ll r$. The unified Newton-type potential (10) leads to real Coulomb forces, $q_{1}\left[-\nabla\left(-q_{2} r_{o}^{-1}\right) \ln \left(1+r_{o} r^{-1}\right)\right]=i e_{1}\left(-i e_{2}\right) \hat{\mathbf{r}} / r\left(r+r_{o}\right) \approx e_{1} e_{2} \hat{\mathbf{r}} / r^{2}$, with mutual repulsion of like electric charges $e_{1}$ and $e_{2}$, and attraction of unlike ones. The radiation selfforce, $f_{\text {rad }}=2 q^{2} \ddot{\mathbf{v}} / 3 c^{3}=2\left(-i e_{o}\right)^{2} \ddot{\mathbf{v}} / 3 c^{3}=-2 e_{o}^{2} \ddot{\mathbf{v}} / 3 c^{3}$, takes the physical, damping direction for the imaginary charge, contrary to a mathematical self-acceleration of the real electric charge.

The gravitational sign in the Poisson equation for imaginary electric energies suggests to inverse positive and negative imaginary densities of electric charges, $\rho \rightarrow-\rho$, in the Maxwell-Lorentz equations (1), which are irrelevant to the conventional signs of electrons and protons. The electrostatic solution for charged imaginary densities in (1) with the gravitational type direction of the radial Coulomb 
field, $\vec{D}(\mathbf{x})=-Q \hat{\mathbf{x}} /|\mathbf{x}|\left(|\mathbf{x}|+Q \varphi_{o}^{-1}\right)$, facilitates the unification of gravity and electricity on the basis of one complex energy charge $Q \varphi_{o} \equiv\left(q_{m}+i q_{e}\right) c^{2} / \sqrt{G} \Rightarrow(\sqrt{G} m+i e) c^{2} / \sqrt{G}$. The Maxwell-Lorentz equations (1) for the massless elementary charge can be extended on complex energy flows of the radial carrier with the elementary charge density $\rho(\mathbf{r}, t) \equiv(\sqrt{G} m+i e) n(\mathbf{x}, t)=-\operatorname{div} \vec{D}(\mathbf{x}, t) / 4 \pi$,

$$
\left\{\begin{array}{l}
\operatorname{div} \vec{B}(\mathbf{x}, t) \varphi_{o}=0 \\
\operatorname{ccurl} \vec{B}(\mathbf{x}, t) \varphi_{o}=\vec{v} \operatorname{div} \vec{D}(\mathbf{x}, t) \varphi_{o}+\partial_{t} \vec{D}(\mathbf{x}, t) \varphi_{o} \\
\operatorname{ccurl} \vec{D}(\mathbf{x}, t) \varphi_{o}=-\partial_{t} \vec{B}(\mathbf{x}, t) \varphi_{o} .
\end{array}\right.
$$

One may indeed remove from the Maxwell-Lorentz system of equations for the electron its density $\rho(\mathbf{r}, t)$, which is the function of fields in non-dual physics rather than an independent entity in the empty space paradigm.

The unified gravi-electric potential of the static system of complex charges $q_{k}=\sqrt{G} m_{k}+i e_{k} \equiv \varphi_{o} z_{k}$ is given by the analytic function,

$$
W(\mathbf{x})=-\frac{c^{2}}{\sqrt{G}} \ln \left(1+\frac{z_{1}}{\left|\mathbf{x}-\mathbf{a}_{1}\right|}+\frac{z_{2}}{\left|\mathbf{x}-\mathbf{a}_{2}\right|}+\ldots+\frac{z_{n}}{\left|\mathbf{x}-\mathbf{a}_{n}\right|}\right),
$$

which is responsible for the Equivalence Principle of passive and active energy densities in each point of the non-empty space plenum,

$$
\epsilon_{p}(\mathbf{x}) \equiv \frac{[-\nabla W(\mathbf{x})]^{2}}{4 \pi}=\frac{\varphi_{o} \nabla^{2} W(\mathbf{x})}{4 \pi} \equiv \epsilon_{a}(\mathbf{x})
$$

The volume energy integral of overlapping material spaces with paired interactions demonstrates the system energy conservation due to interference exchanges $2 a b$ in join densities $(a+b)^{2}$,

$$
\mathcal{E}_{s y s} \equiv \int \epsilon_{p}(\mathbf{x}) c^{2} d^{3} x=\frac{\varphi_{o}^{2}}{4 \pi} \int\left(\frac{\frac{\left(\mathbf{x}-\mathbf{a}_{1}\right) z_{1}}{\left|\mathbf{x}-\mathbf{a}_{1}\right|^{3}}+\frac{\left(\mathbf{x}-\mathbf{a}_{2}\right) z_{2}}{\left|\mathbf{x}-\mathbf{a}_{2}\right|^{3}}+\ldots+\frac{\left(\mathbf{x}-\mathbf{a}_{n}\right) z_{n}}{\left|\mathbf{x}-\mathbf{a}_{n}\right|^{3}}}{1+\frac{z_{1}}{\left|\mathbf{x}-\mathbf{a}_{1}\right|}+\frac{z_{2}}{\left|\mathbf{x}-\mathbf{a}_{2}\right|}+\ldots+\frac{z_{n}}{\left|\mathbf{x}-\mathbf{a}_{n}\right|}}\right)^{2} d^{3} x \equiv \varphi_{o}^{2} \sum_{k=1}^{n} z_{k} \equiv \sum_{k=1}^{n} \mathcal{E}_{k},
$$

and the system energy compensation by self-interaction of charged densities in the potential (12),

$$
\frac{\varphi_{o}}{4 \pi} \int\left(\frac{\frac{\left(\mathbf{x}-\mathbf{a}_{1}\right) z_{1}}{\left|\mathbf{x}-\mathbf{a}_{1}\right|^{3}}+\frac{\left(\mathbf{x}-\mathbf{a}_{2}\right) z_{2}}{\left|\mathbf{x}-\mathbf{a}_{2}\right|^{3}}+\ldots+\frac{\left(\mathbf{x}-\mathbf{a}_{n}\right) z_{n}}{\left|\mathbf{x}-\mathbf{n}_{n}\right|^{3}}}{1+\frac{z_{1}}{\left|\mathbf{x}-\mathbf{a}_{1}\right|}+\frac{z_{2}}{\left|\mathbf{x}-\mathbf{a}_{2}\right|}+\ldots+\frac{z_{n}}{\left|\mathbf{x}-\mathbf{a}_{n}\right|}}\right)^{2}\left[1-\ln \left(1+\frac{z_{1}}{\left|\mathbf{x}-\mathbf{a}_{1}\right|}+\frac{z_{2}}{\left|\mathbf{x}-\mathbf{a}_{2}\right|}+\ldots+\frac{z_{n}}{\left|\mathbf{x}-\mathbf{a}_{n}\right|}\right)\right] d^{3} x \equiv 0
$$

\section{Gravitation repulsion by dense mass-energy fields}

For a pure mechanical system, for example, the volume energy (14) of nonempty space at $z_{k} \Rightarrow$ $G m_{k} / c^{2} \equiv r_{k}$ can be represented analytically at $r_{k}+r_{l} \ll\left|\mathbf{a}_{k}-\mathbf{a}_{l}\right|$ as a sum of GR metric energy,

$$
\begin{gathered}
\mathcal{E}_{G R} \approx \int \frac{\varphi_{o}^{2} r_{1}^{2} d^{3} x}{4 \pi|\mathbf{x}|^{4}\left(1+\frac{r_{1}}{|\mathbf{x}|}+\frac{r_{2}}{\left|\mathbf{a}_{1}-\mathbf{a}_{2}\right|}+\ldots+\frac{r_{n}}{\mid \mathbf{a}_{1}-\mathbf{a}_{n}}\right)^{2}}+\int \frac{\varphi_{o}^{2} r_{2}^{2} d^{3} x}{4 \pi|\mathbf{x}|^{4}\left(1+\frac{r_{1}}{\left|\mathbf{a}_{2}-\mathbf{a}_{1}\right|}+\frac{r_{2}}{|\mathbf{x}|}+\ldots+\frac{r_{n}}{\left|\mathbf{a}_{2}-\mathbf{a}_{n}\right|}\right)^{2}} \\
+\ldots+\int \frac{\varphi_{o}^{2} r_{n}^{2} d^{3} x}{4 \pi|\mathbf{x}|^{4}\left(1+\frac{r_{1}}{\left|\mathbf{a}_{n}-\mathbf{a}_{1}\right|}+\frac{r_{2}}{\left|\mathbf{a}_{n}-\mathbf{a}_{2}\right|}+\ldots+\frac{r_{n}}{|\mathbf{x}|}\right)^{2}}=\sum_{i=1}^{n} \mathcal{E}_{i} \sqrt{g_{o o}^{\neq i}\left(\mathbf{a}_{i}\right)} \approx \sum_{i=1}^{n}\left(m_{i} c^{2}-m_{i} \sum_{k \neq i}^{n} \frac{G m_{k}}{R_{i k}}\right)>0,
\end{gathered}
$$

with $E_{2} \equiv m_{2} c^{2} \sqrt{g_{o o}^{\neq 2}\left(\mathbf{a}_{2}\right)} \equiv m_{2} c^{2} /\left(1+\frac{r_{1}}{\left|\mathbf{a}_{2}-\mathbf{a}_{1}\right|}+\frac{r_{3}}{\left|\mathbf{a}_{2}-\mathbf{a}_{3}\right|}+\ldots+\frac{r_{n}}{\left|\mathbf{a}_{2}-\mathbf{a}_{n}\right|}\right)$, for instance, and the interference (dark) part of the system energy,

$$
E_{\text {dark }}=\frac{\varphi_{o}^{2}}{4 \pi} \sum_{i=1}^{n} \sum_{k \neq i}^{n} \int_{0}^{2 \pi} d \varphi \int_{0}^{\infty} r^{2} d r \int_{0}^{\pi r_{i} r_{k}\left(r^{2}-R_{i k} r \cos \theta\right) \sin \theta d \theta} \frac{r^{3}\left(R_{i k}^{2}+r^{2}-2 R_{i k} r \cos \theta\right)^{3 / 2}}{r_{i=1}^{n}}\left(m_{i} \sum_{k \neq i}^{n} \frac{G m_{k}}{R_{i k}}\right)>0 .
$$


Any mechanical system tends to an equipartition distribution of energy between available degrees of freedom that results from (16)-(17) to Newtonian gravitational attraction (16) in week fields with low dark energy (17), but to gravitational repulsion in dense energies with an excess of the dark component. Indeed, the radial fall speed $d r / d t= \pm c \sqrt{g_{o o}\left(1-g_{o o}\right)}$ can be found from the GR interval equation $(d r / d s)^{2}+(r d \varphi / d s)^{2}-E_{m}^{2} / m^{2} g_{o o}=-1$ at $E_{m} / m \equiv c^{2} \sqrt{g_{o o}} / \sqrt{1-v^{2} c^{2}}=$ const $\Rightarrow 1$ and $d \varphi / d s=0, d s=\sqrt{g_{o o}} c d t \sqrt{1-v^{2} c^{-2}}, v^{2}=\left(d r / \sqrt{g_{o o}} d t\right)^{2}$. Then, coordinate time acceleration $d^{2} r / d t^{2}$ in the flat space metric (7) with $g_{o o}(r)=r^{2} /\left(r+r_{o}\right)^{2}$,

$$
d^{2} \mathbf{r} / d t^{2}=-c^{2} r_{o} \mathbf{r}\left(r^{2}-2 r_{o} r-r_{o}^{2}\right) /\left(r+r_{o}\right)^{5},
$$

universally describes both the Newton attraction $-r_{o} c^{2} \mathbf{r} / r^{3}$ for $r_{o} \ll r$ and the strong field GR repulsion $+\mathbf{r} c^{2} / r_{o}^{2}$ for $r \ll r_{o} \equiv G M / c^{2}$.

The radial dimension of the Metagalaxy is less than its gravitational scale $R_{o}=G M_{\text {Meta }} / c^{2}$ as is widely accepted. According to (18), this dense Metagalaxy should repeal its material elements behind $R_{o}(1+\sqrt{2})$. The strong-field limit, when $r \ll r_{o}$ and $d r / d t \approx c r / R_{o} \Rightarrow r H_{o}$, corresponds to the measured Hubble velocities of expanding galaxies at $R_{o} \Rightarrow c / H_{o}=1,3 \times 10^{26} \mathrm{~m}$ that computes $M_{\text {Meta }}=R_{o} c^{2} / G=1.8 \times 10^{53} \mathrm{~kg}$ very close to direct observations. Acceleration of the Universe expansion in the dense mass-energy limit, $d^{2} r / d t^{2}=c^{2} r / R_{o}^{2} \Rightarrow r H_{o}^{2}$, is proportional to the distance $r$ that redefine the Hubble velocity as $V_{H}=r f(t)=r H_{o}\left(1+H_{o} t\right)$.

\section{Maxwell equations are local energy identities in non-dual physics}

By pairing locally energy densities of the radial field and the continuous particle, one also unifies in (10) gravi-mechanical and electric self-energies $q \varphi_{o} \Rightarrow Q \varphi_{o}=(\sqrt{G} m+i e) c^{2} / \sqrt{G}$. Such a double unification (particle with field and gravity with electricity that was predicted many years ago [2]) assumes the application of the complex charge $q \Rightarrow Q=(\sqrt{G} m+i e)$ in the logarithmic potential (10) for non-empty space. The sphere of the microscopic radius $\left|r_{o}\right|=\left|Q / \varphi_{o}\right|$ contains exactly half of the complex electron charge $Q$. The other half of the elementary astrocharge is distributed over micro, macro, and mega scales in the Universe, which is already known [8] as the non-local material system.

The electron's density scale $\left|r_{o}\right| \approx e_{o} \sqrt{G} / c^{2}=1,38 \times 10^{-34} \mathrm{~cm}$ is even less than the Planck's length, $l_{p} \equiv \sqrt{\hbar c}\left(\sqrt{G} / c^{2}\right)=1,62 \times 10^{-33} \mathrm{~cm}$, and surely is much smaller of the current limit $10^{-17} \mathrm{~cm}$ for space inhomogeneity measurements. The ratio of classical and quantum scales, $\left|r_{o}\right|^{2} / l_{p}^{2} \equiv \alpha \approx 1 / 137$, can shed some light on the physical origin of the Sommerfeld (fine-structure) constant. The very small spatial scale $10^{-34} \mathrm{~cm}$ for main densities of the radial electron explains the formal success of the $\delta$ operator modeling of the nonlocal energy carrier by the point matter approximation. The formal interaction of such point-like concentrations of energy with external weak distributions of energy is conceptually incorrect for extended charges due to their global overlap in the entire Universe.

One can verify quantitatively that the complex astrocharge distribution, $\rho(r)=Q n(r)=(\sqrt{G} m+$ ie) $n(r)$ with $r_{o}=Q \sqrt{G} / c^{2}$, generates the potential (10) under the regular integral rule for classical gravitation and electrodynamics,

$$
\begin{aligned}
& \frac{(-Q)}{r_{o}} \ln \left(1+\frac{r_{o}}{r}\right)=\int \frac{(-Q) n\left(r^{\prime}\right) d v^{\prime}}{\left|\vec{r}-\vec{r}^{\prime}\right|}=-\int_{o}^{\infty} \int_{-\pi / 2}^{\pi / 2} \int_{o}^{2 \pi} \frac{d \phi^{\prime} \sin \theta^{\prime} d \theta^{\prime} r^{\prime 2} d r^{\prime}}{\sqrt{r^{2}+r^{\prime 2}-2 r r^{\prime} \cos \theta^{\prime}}} \frac{Q r_{o}}{4 \pi r^{\prime 2}\left(r^{\prime}+r_{o}\right)^{2}} \\
= & -\int_{o}^{\infty} \frac{d r^{\prime} Q r_{o}}{\left(r^{\prime}+r_{o}\right)^{2}}\left(\frac{\left|r^{\prime}+r\right|-\left|r^{\prime}-r\right|}{2 r r^{\prime}}\right)=-\int_{r}^{\infty} \frac{Q d r^{\prime}}{r_{o}}\left(\frac{1}{r^{\prime}}-\frac{1}{r^{\prime}+r_{o}}\right)=\int_{r}^{\infty} \hat{\mathbf{r}}^{\prime} \vec{D}\left(r^{\prime}\right) d r^{\prime}=W(r) .
\end{aligned}
$$

Notice that the potential $W(r)$ coincides with the work associated with the replacement of a unit probe (positive) charge from the point $r$ to $\infty$ against the radial field $\hat{\mathbf{r}}^{\prime} \vec{D}\left(r^{\prime}\right)=D(r)=-\partial_{r} W(r)$. The 
integration over $r^{\prime}$ within $0 \leq r^{\prime} \leq r$ vanishes identically in (19) in agreement with the physical meaning of potentials for a probe body.

Like the first pair of Maxwell field identities, $\nabla_{\lambda} F_{\mu \nu}+\nabla_{\mu} F_{\nu \lambda}+\nabla_{v} F_{\lambda \mu} \equiv 0$, the second pair of Maxwell current equations can also be considered (in the non-empty space paradigm) as identities for balanced momentums $\rho \varphi_{o} u^{\mu} / c$ of (yin-yang) paired energy densities,

$$
\left\{\begin{array}{l}
{\left[\nabla_{\lambda} F_{\mu \nu}(x)+\nabla_{\mu} F_{v \lambda}(x)+\nabla_{v} F_{\lambda \mu}(x)\right] \varphi_{o} / 4 \pi c \equiv 0} \\
{\left[\delta_{\lambda}^{\mu}-u^{\mu}(x) u_{\lambda}(x)\right] \nabla_{\nu} F^{\nu \lambda}(x) \varphi_{o} / 4 \pi c \equiv 0 .}
\end{array}\right.
$$

Here we used the following identical relations for classical fields:

$$
\left\{\begin{array}{l}
\nabla_{v} F^{\nu \mu}(x) \equiv 4 \pi j^{\mu}(x) / c \equiv 4 \pi \rho(x) u^{\mu}(x) \\
u_{\mu}(x) \nabla_{v} F^{\nu \mu}(x) \equiv 4 \pi \rho(x) \\
\nabla_{v} F^{\nu \mu}(x) \equiv u^{\mu}(x) u_{\lambda}(x) \nabla_{v} F^{\nu \lambda}(x) .
\end{array}\right.
$$

The energy-momentum balance of paired 4-vector flows in the second identity (20) clams the absence of curl energy currents along directions normal to the 4 velocity $u^{\mu}$ of field-energy densities, where $u_{\mu} u^{\mu}=1$. These is no much sense to introduce particles at all in the non-dual scheme for fieldenergy identities (20). In other words, material reality is nothing but inhomogeneous energy flows through all spatial points of the Universe.

Once one identified electric currents with field-energy flows in the Maxwell-Lorentz theory for extended electrons, the variation techniques for non-dual material fields should be different from textbook variation procedures of the empty space physics. Indeed, the current Maxwell equations were derived in the dual approach from the summary action of fields and particles varied and fixed independently, like $\delta\left(A_{\mu} j^{\mu}\right)=j^{\mu} \delta A_{\mu}$. In non-dual physics, variations of self-energy of material flows in their potentials $\delta\left(A_{\mu} j^{\mu}\right)$ are equal to the sum $j^{\mu} \delta A_{\mu}+A_{\mu} \delta j^{\mu}$, with the double variational result

$$
\delta\left(c A_{\mu} \nabla_{v} F^{v \mu} / 4 \pi\right)=(c / 4 \pi)\left[\delta A_{\mu} \nabla_{v} F^{\nu \mu}+A^{\mu} \nabla^{v}\left(\partial_{\mu} \delta A_{v}-\partial_{v} \delta A_{\mu}\right)\right] \Rightarrow(c / 2 \pi) \nabla_{v} F^{v \mu} \delta A_{\mu}=2 j^{\mu} \delta A_{\mu} .
$$

Therefore, the Lagrangian for non-dual charged energies should posses double electromagnetic densities $F_{\mu \nu} F^{\mu v}$ in the non-empty space action,

$$
S_{n-e m}=-\frac{1}{c} \int d \Omega\left[\frac{1}{c} A_{\mu} j^{\mu}+\frac{1}{8 \pi} F_{\mu \nu} F^{\mu v}\right],
$$

compared to the dual (field + matter) approach. Then the variations of (23) with respect to four field potentials $A_{\mu}$ result in the Maxwell relation $4 \pi j^{\mu} / c=\nabla_{v} F^{\nu \mu}$ or energy flow identities in (20) which is the Lagrange equation of motion. It is worth to note, that the non-empty space action (23) vanished on the Lagrange variation trajectories:

$$
\begin{aligned}
S_{n-e m}=-\frac{1}{c} \int d \Omega\left[\frac{1}{c} A_{\mu} j^{\mu}\right. & \left.+\frac{1}{8 \pi} F_{\mu \nu} F^{\mu \nu}\right]=-\int \frac{d \Omega}{8 \pi c}\left[2 A_{\mu} \nabla_{v} F^{\nu \mu}+\left(\partial_{\mu} A_{v}-\partial_{v} A_{\mu}\right) F^{\mu v}\right] \\
& =-\int \frac{d \Omega}{4 \pi c} A_{\mu} \nabla_{v}\left(F^{\nu \mu}-F^{\mu \nu}\right)-\oint \frac{d S_{\mu}}{4 \pi c} A_{v} F^{\mu v}=0+0=0 .
\end{aligned}
$$

In other words, self interactions of extended charges in electrodynamics balance their electric selfenergies like gravitational self-interactions in (8) balance mechanical self-energies $m c^{2}$ of elementary mass-energy carriers. This is an expected result that motion along Lagrange trajectories, like (20), nullifies the action integral. Recall that the conventional current+field electromagnetic action in dual physics with $F_{\mu \nu} F^{\mu \nu} / 16 \pi$ instead of $F_{\mu \nu} F^{\mu \nu} / 8 \pi$ does not match such a path nullification in the Lagrange variation formalism. 


\section{Inward and outward longitudinal waves within the continuous electron}

Sphere radial currents or moving electric densities under strict spherical symmetry do not generate magnetic fields in Maxwell's electrodynamics as is well known. Such magnetic-field-free currents may equally have inward and outward directions with respect to a center of the radial electron. Timevarying currents of charged densities should be balanced by time-varying Maxwell displacement currents. In other words, the static radial shape of the elementary charge (associated with its static Coulomb field) may be accompanied by radial wave modulations with longitudinal electric induction.

Periodical inward, $\left(-\hat{\mathbf{r}} V_{l}\right) \rho_{\text {in }}(r, t)$, and outward, $\hat{\mathbf{r}} V_{l} \rho_{\text {out }}(r, t)$, currents, where $\hat{\mathbf{r}} \equiv \vec{r} / r$, are balanced by radial displacement currents $\partial_{t} d_{i n}(r, t) \hat{\mathbf{r}}$ and $\partial_{t} d_{\text {out }}(r, t) \hat{\mathbf{r}}$, respectively, with $\operatorname{div}\left[d_{i n}(r, t) \hat{\mathbf{r}}\right]=$ $4 \pi \rho_{\text {in }}(r, t)$ and $\operatorname{div}\left[d_{\text {out }}(r, t) \hat{\mathbf{r}}\right]=4 \pi \rho_{\text {out }}(r, t)$ :

$$
\left\{V_{l} \partial_{r} r^{2}\left[d_{\text {out }}(r, t)-d_{\text {in }}(r, t)\right]+r^{2} \partial_{t}\left[d_{\text {out }}(r, t)+d_{\text {in }}(r, t)\right]\right\} \hat{\mathbf{r}} / r^{2}=0 .
$$

This classical equation for balanced radial currents with the spherical symmetry has wave solutions along both outward and inward directions,

$$
\left\{\begin{array}{l}
\vec{d}_{\text {out }}(r, t)=\hat{\mathbf{r}} q a_{\text {out }} \cos \left(\omega t-k r+\varphi_{\text {out }}\right) / r^{2} \\
\vec{d}_{\text {in }}(r, t)=-\hat{\mathbf{r}} q a_{\text {in }} \cos \left(\omega t+k r+\varphi_{\text {in }}\right) / r^{2}
\end{array}\right.
$$

These wave modulations with $k=\omega / V_{l}$ are responsible for dynamical reshaping of the continuous elementary charge $q$ in external fields. Such longitudinal waves within the elementary charged continuum keep its energy, while transverse electromagnetic waves are responsible for energy exchanges between different carriers of energy. In general, the dimensionless wave amplitudes $a_{\text {out }}$ and $a_{\text {in }}$ may have different complex values related to power of longitudinal waves within the extended electron. However, there are no internal power flows within the elementary continuum in equilibrium. In this case, superposition of outward and inward waves (26) can exist as a standing wave,

$$
\vec{d}_{s t a n}(r, t)=\frac{q a \hat{\mathbf{r}}}{r^{2}}[\cos (\omega t-k r)-\cos (\omega t+k r)]=\frac{2 q a \hat{\mathbf{r}}}{r^{2}} \sin k r \cdot \sin \omega t,
$$

with its time-averaged energy integral over the elementary charge volume,

$$
E_{a, \omega}=\int_{o}^{\infty} \frac{(2 q a)^{2} \sin ^{2} k r<\sin ^{2} \omega t>_{t}}{4 \pi r^{4}} 4 \pi r^{2} d r=2 q^{2} a^{2} \int_{o}^{\infty} d r \frac{\sin ^{2} k r}{r^{2}}=\pi a^{2} q^{2} \frac{\omega}{V_{l}} .
$$

A set of internal standing waves (27) with different frequencies $\omega$ and amplitudes $a$ may play in Maxwell's electrodynamics the same role as the foam of virtual photons around to the quantum electron in wave mechanics. De Broglie was first who assigned in 1923 the internal wave process to each elementary particle with inertia. And later he specified this wave process for his unique physical interpretation of quantum phenomena [10]. We tend to support the internal wave process in therms of radial wave modulations (26)-(28) which shed some "classical' light on the celebrated doubleslit experiment. In fact, not only quantum mechanics but also Maxwell's theory of classical electric fields and electric energy flows may comment on the wave motion of continuous electrons. One day classical and quantum electrodynamics will lead to similar wave physics for the elementary charge.

\section{Conclusions}

Unified electric currents and mass flows of the elementary continuous carrier are proportional to electric energy and mas-energy flows, respectively, of this carrier. The radial Maxwell-Lorentz electron 
(1)-(4) tends to replicate (astro)distributions of the quantum electron in the De Broglie - Bohm formulation of wave mechanics [9]. In particular, De Broglie periodical oscillations, $\psi=\psi_{o}$ expi $(\omega t-\mathbf{k x})$, of elementary particle's matter everywhere in the Universe may be associated in the developed classical approach to internal wave modulations (26) - (27) within the continuous electron.

Each radial electron possesses a fine, but finite, density in all spatial points. Information free elastic reshaping of these elementary densities might occur with internal longitudinal waves without energy losses/gains, while free transverse waves and other dissipative signals (with information and mutual energy exchanges between overlapping microcosms of elementary energies) obey the speed restriction of Special Relativity regarding centers of radial carriers.

The experimental result [1] for the rigid motion of Coulomb's fields confirms the non-empty paradigm for overlapping material bodies. In non-dual physical reality, nebular dense fronts of infinitely extended masses (observed as the planets) are moving within, not outside, the $r^{-4}$ mass-energy distribution of the radial Sun. Such a global overlap of locally undivided continuous carriers of massenergy in joint non-empty space explains not only the electromagnetic laboratory experiment [1], but also the gravitational Laplace problem of the Solar system stability.

Outward spherical waves were considered in the conventional empty space physics as retarded waves which have already left their point source. Inward spherical waves were called non-physical waves by the dual model, because these (advanced) waves have not yet met their point source in question. The non-empty space paradigm for radial material sources provides equal physical rights to outward and inward waves in reality of non-local bodies. In this way, intercepted energy flows along advanced directions toward moving centers of cosmic bodies, as was claimed by astrophysicists N. Kozyrev [11], may be considered as a justification of the non-empty space in reality. Similarly, inward radial signals might be expected under acceleration of electric charges when their radial structures attain external energy. It appears that an interesting development of experiments [1] might be their reiteration with proton beams at the Large Hadron Collider. In general, wave energy flows along a computed advanced axis toward moving centers of radial energies may ultimately justify the nonempty space paradigm for non-dual reality of the nonlocal world.

\section{References}

[1] R. de Sangro, G. Finocchiaro, P. Patteri, M. Piccolo, G. Pizzella, Measuring propagation speed of Coulomb fields, Eur. Phys. J. C 75, 137 (2015)

[2] M.-A. Tonnelat, The principles of electromagnetic theory and relativity (Riedel Publishing Co., Dordrecht, 1966)

[3] L. de Broglie, New perspectives in physics (Basic Books, New York, 1962)

[4] G. Mie, Ann. der Physik 37, 511 (1912); ibid 39, 1 (1912); ibid 40, 1 (1913)

[5] J. Schwinger, in Quantum, Space and Time - The Quest Continues (Ed. A.O. Barut, A. Van der Merwe, and J.-P. Vigier, Cambridge University Press, 1984) p.620

[6] H.A. Lorentz, The Theory of Electrons (Leipzig, 1916)

[7] I.E. Bulyzhenkov, Int. Jour. of Theor. Phys. 47, 1261 (2008); Pure field electrodynamics of continuous complex charges: 4th year course tutorial (MIPT, Moscow, 2015) ISBN 978-5-7417-0554-4

[8] A. Aspect, P. Grangier, G. Roger, Phys. Rev. Let. 47, 460 (1981), ibid 49, 91 (1982)

[9] D. Bohm, Phys. Rev. 85, 166 (1952); D. Bohm and B.J. Hiley, The Undivided Universe, Routledge, London, 1993

[10] L. de Broglie, Reinterpretation of Wave Mechanics (Gauthier-Villars, Paris, 1971)

[11] N.A. Kozyrev, Selected Works (St-Petersburg State University, St-Petersburg, 1991, in Russian) 